\title{
LOAD BEARING ANALYSIS ON LUMBOSACRAL DISC IN PRE-OPERATIVE AND POST-OPERATIVE THORACIC SCOLIOSIS PATIENT
}

\section{Mohankumar Palaniswamy ${ }^{1,}$, , Anis Suhaila Shuib ${ }^{2}$, Shajan Koshy ${ }^{2}$}

\author{
${ }^{1}$ Affiliation 1. \\ Physiotherapist, Chennai, 600032, Tamil Nadu, India. \\ ${ }^{2}$ Affiliation 2. \\ Taylor's University, Subang Jaya 47500, Selangor, Malaysia. \\ *Corresponding Author's Email: ${ }^{1}$ mohanphysionix06@live.com \\ Article History: Received May 30, 2021; Revised June 25, 2021; \\ Accepted June 28, 2021
}

\begin{abstract}
Scoliosis is a musculoskeletal disorder seen all around the world. It affects both the alignment of the vertebra and intervertebral disc. Scoliosis can be treated conservatively with a cast and brace or surgically with spinal instrumentation. During planning for surgical instrumentation, several factors need to be considered. Among those, biomechanical changes in the non-scoliotic vertebrae and discs are important. This is vital in determining the future degenerative changes of the spine. For this reason, this study was conducted with a finite element model of the lumbosacral joint using CT scan files to find the total deformation and equivalent static strain of the lumbosacral disc between pre and post-operative thoracic scoliosis patients. From the results, it is evident that there is a biomechanical change in the lumbosacral disc and structural change in the vertebral alignment followed immediately after corrective surgery. The correction in the alignment of the scoliotic spine brings changes to the biomechanical functionality and loadbearing capacity of the lumbosacral intervertebral disc before and after surgery.
\end{abstract}

KEYWORDS: Thoracic scoliosis, Lumbosacral disc, Finite element analysis, Vertebral alignment, Vertebral load.

\subsection{INTRODUCTION}

The human vertebral column consists of 24 articulating and 9 fused vertebrae (7 cervical, 12 thoracics, 5 lumbar, 5 fused sacral, and 4 fused 
coccyx vertebrae), which totals to 33 vertebrae. An intervertebral disc, held together by ligaments, separates the column formed by the articulating vertebra with the adjacent vertebra. This results in the formation of curves during weight-bearing. Scoliosis is a deformity of the vertebral column, in which the spine is abnormally twisted and curved to the sides. Globally scoliosis is one of the most common musculoskeletal disorders affecting the pediatric age group with a prevalence of $2-3 \%$. When examining the spine from the back, a scoliotic spine will appear ' $C$ ' shaped (single curvature) or ' $S$ ' shaped (double curvature). It can be treated conservatively with a cast or brace or surgically with spinal instrumentation. Opting for surgical intervention depends upon various factors such as etiology, severity, cardiopulmonary involvement, Cobb's angle, and age. During spinal instrumentation surgery, surgeons fuse the scoliotic vertebrae using metal implants. Since the alignment of vertebrae is affected in scoliosis, the Center of Gravity ( $\mathrm{CoG}$ ) and Line of Gravity ( $\mathrm{LoG}$ ) is also affected. In an anatomical standing posture, the CoG and LoG lie anterior to the sacrum bone (S2). Whereas, in scoliosis patients, the CoG and LoG are deviated depending on the configuration of the spine. This level of deviation can be roughly understood by measuring the coronal balance, sagittal balance, thoracic trunk shift, thoracolumbar and lumbar sagittal alignment.

A study done by Damavandi et al., [1] on the head and trunk mass, and center of mass position estimations in able-bodied and scoliotic girls concluded that the pre-operative scoliotic girls have greater pelvic forward tilt and trunk inclination compared to normal subjects. Whereas, another study done by Park et al., [2] on the effect of scoliosis angle on sway on the center of gravity found that the whole-body balancing ability in pre-operative scoliosis patients is significantly different from normal persons. This explains the importance of pelvic tilt, the center of gravity, and trunk inclination in maintaining a proper posture. In scoliosis patients, since the trunk posture is altered or shifted to one side, it in turn provides more pressure to the lumbar region on the side of the scoliosis curve. Using image processing, a study conducted by Hajizadeh et al., [3] on a 3D multibody model of the scoliotic spine with lateral bending motion for comparison of ribcage flexibility concluded that the load at lumbar joints in the scoliosis model were higher when compared to the normal subjects. The vertebral column is connected with the pelvis and lower limbs forming a continuous plane for weight transmission and support for the body in bipedal gait and posture of humans. The pelvis is made up 
of the hip bones and sacrum. The femur bone of the thigh forms an articulation with the acetabulum to form the hip joint. Weight from the head, arm, and trunk are passed through the vertebral column and transferred to the lower limbs through the sacrum and sacroiliac joint as shown in Figure 1. Intervertebral discs play a major role in transferring load from one vertebra to another. It acts as a shock absorber.

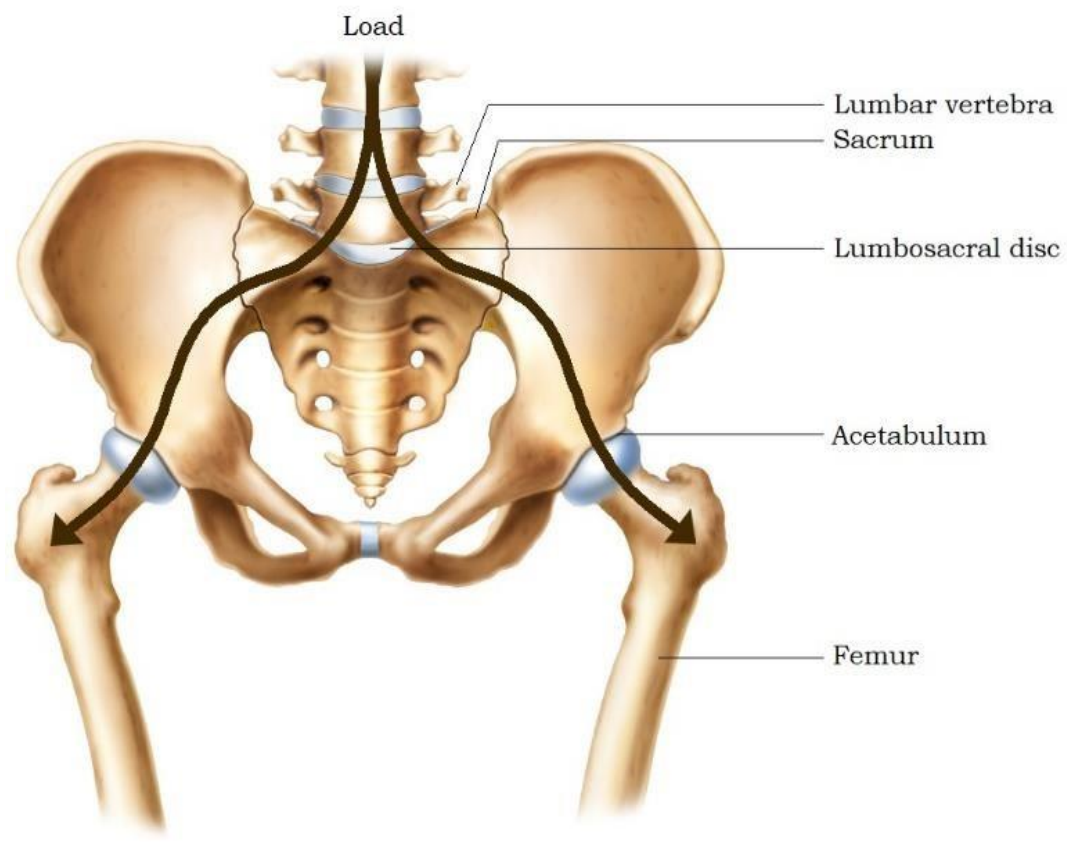

Figure 1: Weight distribution [4].

Pressure within the center of the disc is never zero, because of the pre-existing tension even when the disc is unloaded. The physical property of the intervertebral disc allows it to withstand a considerable amount of load, even when the load is applied for an extended period. A normal intervertebral disc is an anisotropic structure [5]. National Institute for Occupational Safety and Health (NIOSH) guidelines for manual (1994) lifting concluded that the joint between the fifth lumbar vertebra $\left(L_{5}\right)$ and first sacral vertebra $\left(S_{1}\right)$ is the joint of greatest lumbar stress during weight lifting. A study done by Pel et al. found that a $20 \%$ reduction in vertical sacroiliac joint shear resulted in a $70 \%$ increase in sacroiliac joint compression force [6]. In normal subjects, the vertebral column is arranged linearly in the frontal plane and angular in the sagittal plane. Although, in a scoliotic spine, the vertebral column is 
arranged angularly in all three planes as it is often accompanied by some amount of lateral twist in the spine (kyphosis). When scoliosis patients undergo corrective surgery, surgeons focus on correcting the vertebrae causing a scoliosis curve. Surgeons first perform osteotomy (removal of bone parts) and realign the vertebral column manually along the course of the expected normal vertebral curve. Using implants like Harrington rods and Pedicle screws which are made up of stainless steel or titanium, surgeons screw the vertebrae to the Harrington rods using Pedicle screws. Implants are to fix the corrected vertebra in its anatomical position and prevent it from scoliotic recurrence. After fixing the vertebrae, a layer of bone cement or local bone graft is applied over the rearranged vertebral column to fuse the corrected scoliotic vertebrae and to form a single fused vertebra. Thus, the scoliotic vertebral column is rearranged to a new or a normal position.

Some claim that most of the curve is reduced because of the manual pressure. On the other hand, some claim it's due to vertebral fusion and metal implants. A study done by Trobisch et al., [7] on postoperative trunk shift in Lenke 1 and 2 curves concluded that the postoperative trunk shift is common after surgery for adolescent idiopathic scoliosis. But it occurs only in $13.6 \%$ of patients and $65 \%$ of trunk shifts are iatrogenic (caused by the surgeon). If the curve is corrected only by manual pressure, implants can take over the vertebral fusion. Then need for fusing the vertebrae will be a questionable debate. Even after spinal instrumentation, due to the sudden change in the configuration of the spinal column, patients feel a disturbance in their balance. A study by Carvalho de Abreu et al., [8] on the influence of surgical treatment of adolescent idiopathic scoliosis on postural control supports this theory. They concluded that the scoliosis patients have a large CoP oscillation compared to age-matched healthy adults. Even after surgery, oscillation is decreased in the initial 90 days. But later, it remained larger than before surgery. Also, another study done by Nohara et al., [9] on lumbar disc degeneration in patients with adolescent idiopathic scoliosis with spinal fusion claims that $48 \%$ of disc degeneration occurs at the Lumbosacral junction ( $\left.\mathrm{L}_{5}-\mathrm{S}_{1}\right)$ and segments adjacent to fused vertebrae has only $8 \%$ of chance. If the abnormal curve (scoliosis) leads to an altered coronal balance, sagittal balance, thoracic trunk shift, thoracolumbar and lumbar sagittal alignment, then after treating scoliosis, these parameters should, by the right return to normal or at least close to normal. This raises a query of whether scoliotic vertebral fusion also affects the non-scoliotic 
vertebrae (vertebrae not involved in the scoliotic curve) of the spine. Hence, in order to validate this, this study tried to find the total deformation and strain of the lumbosacral intervertebral disc between the pre and post-operated thoracic scoliosis patients. The objectives of this study were to find whether the manual correction during corrective surgery bring any immediate changes to the structure of the spine and to find whether the correction in the alignment of the thoracic scoliotic spine brings any changes to the biomechanical functionality and load-bearing capacity of the lumbosacral intervertebral disc before and after surgery.

\subsection{METHODOLOGY}

The prevalent of scoliosis is 1 to $2 \%$ in adolescence. Among those 1 to $2 \%$, determining the patients with the same side and the same level of scoliosis who underwent corrective surgery is tiresome work and the availability of such kind of data is exceptional. Hence, this study was an observational cross-sectional study. The sampling method followed was convenience sampling.

\subsection{Data Collection}

Data collection was done at Government General Hospital, Chennai, India. Proper consent was obtained from the radiology department before collecting data. Since bone fusion gets complete by 6 to 9 months after surgery [10] [11], pre-operative and post-operative CT scan data of patients who underwent scoliosis correction not more than 2 months and with the age group between 10 to 20 years were selected for this study. Only 1 patient fulfilled the above-mentioned criteria. The patient had right thoracic scoliosis and his lumbar bones had normal vertebral alignment. Radiographs were taken with the patient in a lying position. During standing, patients might tilt their pelvis unknowingly. Both the Pre-operative and post-operative radiographs were obtained in DICOM format. Baseline assessments like age, sex, date of surgery, and date of the scan were noted and presented in Table 1.

Table 1: Patient Details

\begin{tabular}{|c|c|c|c|c|}
\hline Gender & Age & Pre-operative Scan & Date of Surgery & Post-operative Scan \\
\hline M & 19 & $12-01-2016$ & $19-01-2016$ & $26-02-2016$ \\
\hline
\end{tabular}

\subsection{Method}

Obtained DICOM files were imported into an image segmentation software Materialise, version 20.0 (Materialise Inc., Belgium). The 
lumbosacral joint was selected as the region of interest, which consists of the fifth lumbar vertebra (L5), sacrum, and lumbosacral disc. Other parts were cropped out. No metal implants, blood vessels, ligaments, endplates, and muscles were comprised in the image segmentation. Two separate masks were created for bones and discs and were converted into parts. The developed parts were smoothened and wrapped to hide any edges or holes. In order to maintain surface contours, an adaptive remesh was carried out with a triangle edge length of $1 \mathrm{~mm}$. Then, the developed parts were converted into solid volume. This solid volume was meshed with 4 node tetrahedral elements and a maximum edge length of $2 \mathrm{~mm}$, to have a uniformed mesh as shown in Figure 2. The meshed volume of the homogeneous lumbosacral segment was exported to an FEA solver program ANSYS, version 17.2 (Ansys, Inc., U.S.A) as .CDB files. The same procedures were followed for both the pre and post-operative DICOM sets.
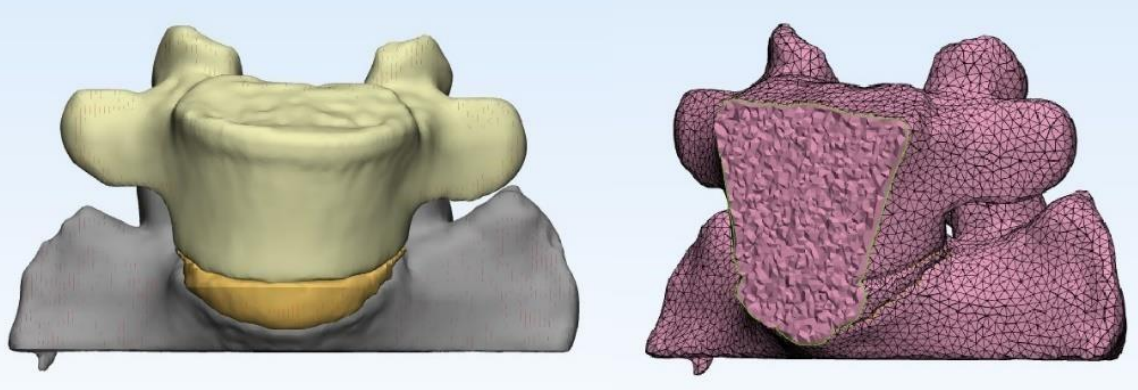

Figure 2: Meshed Lumbosacral Joint

The exported .CDB files were opened with the Ansys Workbench module. Static structural analysis was set to run. A linear homogeneous isotropic material property was used to run the simulation. The isotropic material properties of bone and disc were attained from works of literature [12] and [13] and provided in Table 2. The large deflection was turned off and the direct solver type was selected. In our previous study [14], it was found that on a normal standing position, an average adult weighing $65 \mathrm{kgs}$ with a normal lumbosacral angle of $30^{\circ}$ would exert a net force of $196 \mathrm{~N}$ on the lumbosacral disc. Hence, the same $196 \mathrm{~N}$ force was applied in this model. The sacrum was set as fixed support and $196 \mathrm{~N}$ downward force was applied on the L5 vertebra. The total deformation and equivalent elastic strain of the lumbosacral disc were measured.

Table 2: Material properties of Homogeneous model

\begin{tabular}{|l|l|l|l|}
\hline & Young's Modulus (MPa) & Poisson's Ratio & References \\
\hline
\end{tabular}




\begin{tabular}{|c|c|c|c|}
\hline Bone & 200 & 0.3 & \multirow{2}{*}[12,13]{} \\
\hline Disc & 4 & 0.4999 & {$[109$} \\
\hline
\end{tabular}

\subsection{RESULTS AND DISCUSSION}

Results of applying $196 \mathrm{~N}$ force on the lumbosacral disc between pre and post-operative models showed us that there is a difference between them. Before the correction, the total deformation and equivalent elastic strain of the lumbosacral disc were found to be $0.00020242 \mathrm{~m}$ and $0.03153 \mathrm{~m} / \mathrm{m}$. Whereas, after correction and before complete fusion of the scoliotic vertebrae, the total deformation and equivalent elastic strain of the lumbosacral disc was found to be $0.00011567 \mathrm{~m}$ and $0.019186 \mathrm{~m} / \mathrm{m}$. The number of nodes, elements, total deformation, and equivalent elastic strain of pre-operative and post-operative models is represented in Table 3.

Table 3: Results. TD - Total Deformation, EES - Equivalent Elastic Strain

\begin{tabular}{|c|c|c|}
\hline & Pre-operative & Post-operative \\
\hline Nodes & 65755 & 58247 \\
\hline Elements & 385584 & 326962 \\
\hline TD & $0.00020242 \mathrm{~m}$ & $0.00011567 \mathrm{~m}$ \\
\hline EES & $0.03153 \mathrm{~m} / \mathrm{m}$ & $0.019186 \mathrm{~m} / \mathrm{m}$ \\
\hline
\end{tabular}

It was evident that the area of total deformation and area of equivalent elastic strain in pre and post-operative discs had changed. In the pre-operative lumbosacral disc, the maximum area of total deformation was on the anterior aspect of the disc. Much force was concentrated on the anterior and had uneven distribution. Whereas, in post-operative, the maximum area of total deformation was on the anterolateral aspect of the disc and the force of $196 \mathrm{~N}$ was distributed to the disc to a certain extent as shown in Figure 3.

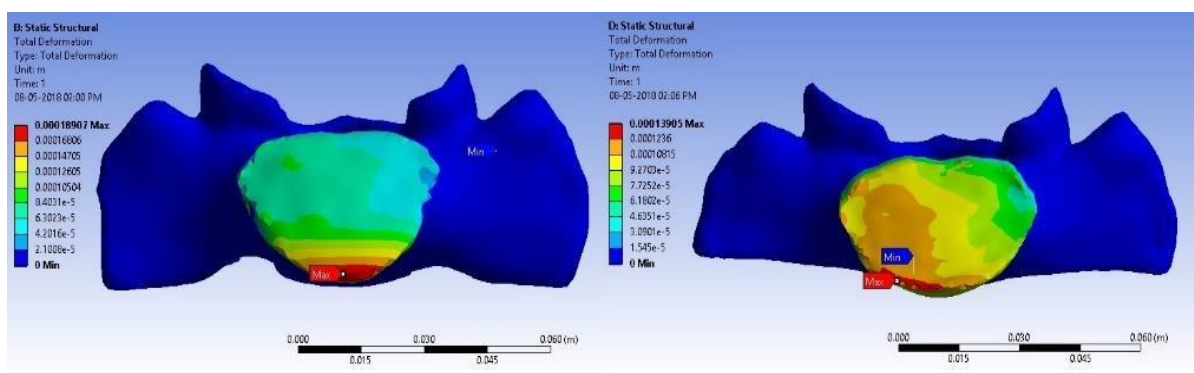

(a)

(b)

Figure 3: Total Deformation of Lumbosacral disc; (a) Pre-operative and 
(b) Post-operative

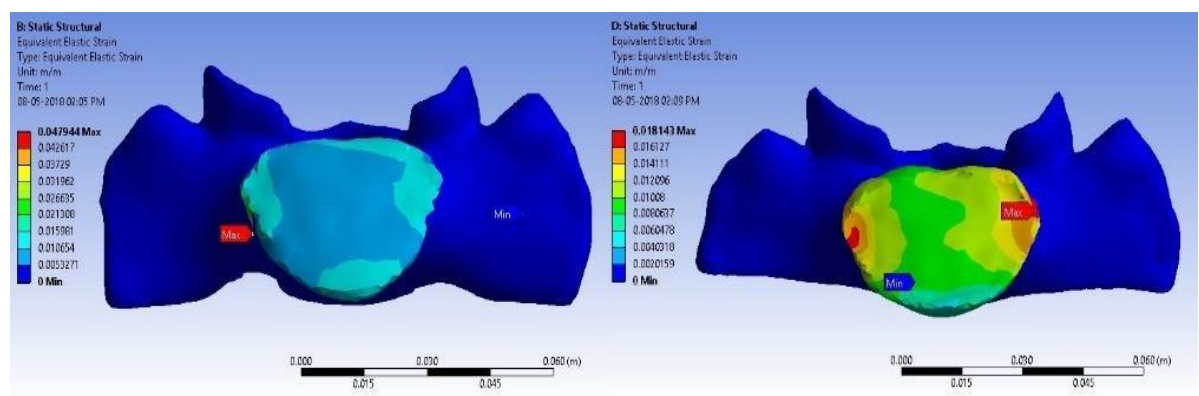

(a)

(b)

Figure 4: Equivalent Elastic Strain of Lumbosacral disc; (a) Preoperative and (b) Post-operative

We noticed the same level of changes in the equivalent elastic strain as well. In pre-operative it was found that the maximum strain was on the lateral aspect of the disc (right side). In post-operative, the maximum strain was found nearly on both the sides (lateral) on top (superior) of the disc as shown in Figure 4. It is also important to note that the number of nodes and elements of the same patient varies between pre and post-operated lumbosacral models.

Earlier, a study by Karami et al., [15] on the assessment of coronal radiographic parameters of the spine in the treatment of adolescent idiopathic scoliosis concluded that precise attention to the coronal balance in pre-operative is vital in the prevention of post-operative decompensation. During the scoliosis correction surgery, surgeons perform osteotomy, place bone grafts in the course of the scoliosis curve, and fuse the vertebrae together. With the help of metal implants, bone grafts, and bone regeneration, curve correction occurs promptly. Another study by Ameri et al., [16] on the natural history of coronal balance after spinal fusion in adolescent idiopathic scoliosis revealed that the first 12 months after posterior spinal fusion is the spontaneous improvement period for coronal balance.

Our earlier study [17] on thoracic trunk shift and coronal balance conducted among 24 pre and post-operated thoracic scoliosis patients found that there is a significant decrease in the trunk shift and coronal balance between pre and post-operative patients. These 24 thoracic scoliosis patients are those who underwent correction surgery at least a year before the date of data collection. Their X-Ray images were used for measurement. The pre-operative thoracic trunk shift and coronal 
balance were measured to be $42.45 \pm 10.36 \mathrm{~mm}$ and $14.75 \pm 4.12 \mathrm{~mm}$. Whereas, the post-operated thoracic trunk shift and coronal balance were measured to be $14.83 \pm 5.18 \mathrm{~mm}$ and $4.25 \pm 1.35 \mathrm{~mm}$. Since the patients had 12 months of duration from the date of surgery to the date of data collection, this decrease in trunk shift and coronal balance could be because of the implants and vertebral bone fusion. Whereas, the present study was conducted with the patient who had only a 1-month duration between the date of surgery and the date of data collection. This indicates that, along with the implants and vertebral bone fusion, the surgeon's manual correction of scoliotic vertebral alignment also plays a major role in scoliosis correction. After correction, the vertebral column gets a new alignment. This forces the rest of the adjacent vertebrae and other bones like ribs and sacrum to regenerate accordingly. This regeneration of bones after scoliosis correction could be the reason for the difference in the number of nodes and elements between the pre and post-operated lumbosacral model of the same patient.

This study had few limitations. Human bone is nonhomogeneous and anisotropic. Since the volume of the lumbosacral segment is small and this study aimed to find only the structural change between pre and post-operative, the property of bone and disc were assumed to be homogeneous and isotropic. The second limitation was the mesh independence study. Instead of performing a mesh independence study to find the optimal mesh size, a standard mesh size of $2 \mathrm{~mm}$ was selected as it was widely used in the literature. It is advisable that the upcoming researchers use more sample size, perform mesh independence study, and consider the model as nonhomogeneous and anisotropic to get more precise results.

\subsection{CONCLUSION}

This study results showed that, the total deformation and equivalent elastic strain of lumbosacral disc at $196 \mathrm{~N}$ force as $0.00020242 \mathrm{~m}$ and $0.03153 \mathrm{~m} / \mathrm{m}$. Whereas, after correction and before complete fusion of the scoliotic vertebrae, it was found to be $0.00011567 \mathrm{~m}$ and 0.019186 $\mathrm{m} / \mathrm{m}$. From this study, it can also be inferred that manual correction in the alignment of the thoracic scoliotic spine during corrective surgery brings immediate changes to the structure of the spine, changes in the disc load distribution pattern, and bone remodeling in the adjacent vertebrae, ribs, and sacrum. This helps in the improvement of the functional capacity of the individual and thus helps in reducing the morbidity due to the deformity before correctional surgery. 


\subsection{ACKNOWLEDGMENTS}

A heartfelt thanks to Dr. Nalli R Yuvaraj, orthopedic spine surgeon from Rajiv Gandhi Government General Hospital, Chennai, for his help and constant support during the data collection. Cordial thanks to Dr. Chin Seong Lim, Faculty of Engineering, University of Nottingham Malaysia, for his help and support in performing structural simulations at the University of Nottingham Malaysia.

\subsection{REFERENCES}

[1] M. Damavandi, G. Dalleau, G. Stylianides, C.-H. Rivard and P. Allard, "Head and Trunk mass and center of mass position estimates in ablebodied and scoliotic girls," Medical Engineering \& Physics, vol. 35, no. 11, pp. 1607-1612, 2013. DOI: 10.1016/j.medengphy.2013.05.010

[2] J.-Y. Park, G. D. Park, S.-G. Lee and J.-C. Lee, "The Effect of Scoliosis Angle on Center of Gravity Sway," Journal of Physical Therapy Science, vol. 25, no. 12, pp. 1629-1631, 2013. DOI: 10.1589/jpts.25.1629

[3] K. Hajizadeh, I. Gibson and L. G, “Developing a 3D Multi-Body Model of the Scoliotic Spine with Lateral Bending Motion for Comparison of Ribcage Flexibility," International Journal of Advanced Design and Manufacturing Technology, vol. 6, no. 1, pp. 25-32, 2013. URL: http://admt.iaumajlesi.ac.ir/article_534824.html

[4] Dr. Morris. (2015). Pelvic Discomfort in Pregnancy [Online]. Available: http://www.drmorris.com.au/pelvic-discomfort-in-pregnancy/. [Accessed 12 May 2021].

[5] X. Gao, Q. Zhu and W. Gu, "An Anisotropic Multiphysics Model for Intervertebral Disk," Journal of Applied Mechanics, vol. 83, no. 2, pp. 1118, 2016. DOI: 10.1115/1.4031793

[6] J. J. Pel, C. Spoor, A. Pool-Goudzwaard, G. Hoek van Dijke and C. Snijders, "Biomechanical Analysis of Reducing Sacroiliac Joint Shear Load by Optimization of Pelvic Muscle and Ligament Forces," Annals of Biomedical Engineering, vol. 36, no. 3, pp. 415-424, 2008. DOI: 10.1007/s10439-007-9385-8

[7] P. D. Trobisch, A. F. Samdani, J. Pahys and P. J. Cahill, "Postoperative trunk shift in Lenke 1 and 2 curves: how common is it? and analysis of risk factors," European Spine Journal, vol. 20, no. 7, pp. 1137-1140, 2011. DOI: 10.1007/s00586-011-1820-8

[8] D. C. Carvalho de Abreu, M. M. Gomes, H. A. Rocha de Santiago, C. F. P. d. S. Herrero, M. A. Porto and H. L. Aparecido Defino, "What is the influence of surgical treatment of adolescent idiopathic scoliosis on postural control?," Gait Posture, vol. 36, no. 3, pp. 586-590, 2012. DOI: 10.1016/j.gaitpost.2012.05.019 
[9] A. Nohara, N. Kawakami, K. Seki, T. Tsuji, T. Ohara, T. Saito and K. Kawakami, "The Effects of Spinal Fusion on Lumbar Disc Degeneration in Patients with Adolescent Idiopathic Scoliosis: A Minimum 10-Year Follow-Up," Spine Deformity, vol. 3, no. 5, pp. 462-468, 2015. DOI: 10.1016/j.jspd.2015.04.001

[10] E. M. Mannen and D. E. Anderson, "Mechanical testing of the thoracic spine and related implants," in Mechanical Testing of Orthopaedic Implants, Duxford, Woodhead Publishing, 2017, pp. 143-160. DOI: 10.1016/B978-0-08-100286-5.00008-1

[11] T. Shimizu, L. G. Kenke, M. Cerpa, E. Beauchamp, L. Y. Carreon, C. I. Shaffrey, K. M. Cheung and M. G. Fehlings, "A Radiographic Analysis of Lumbar Fusion Status and Instrumentation Failure After Complex Adult Spinal Deformity Surgery With Spinopelvic Fixation: Two-Year Follow-up From the Scoli-Risk-1 Prospective Database," Clinical Spine Surgery, vol. 33, no. 10, pp. 545-552, 2020. DOI: 10.1097/BSD.0000000000001008

[12] H. Li and Z. Wang, "Intervertebral disc biomechanical analysis using the finite element modeling based on medical images," Computerized Medical Imaging and Graphics, vol. 30, no. 6-7, pp. 363-370, 2006. DOI: 10.1016/j.compmedimag.2006.09.004

[13] N. Homma, An Approach to Lumbar Vertebra Biomechanical Analysis Using the Finite Element Modeling Based on CT Images, Rijeka: InTech, 2011. URL:

https://downloadmedicalbooks.files.wordpress.com/2011/08/theory_a nd_applications_of_ct_imaging_and_analysis.pdf

[14] M. Palaniswamy, W. Y. Leong, K. H. Cheah and L. C. Yong, "Engineering concepts in analysing lumbosacral load in post-operative scoliotic patients," Journal of Engineering Science and Technology, vol. 13, no. 4 , pp. 1058-1069, 2018. URL: http://jestec.taylors.edu.my/Vol\%2013\%20issue\%204\%20April\%202018 /13_4_15.pdf

[15] M. Karami, A. Maleki and K. Mazda, "Assessment of Coronal Radiographic Parameters of the Spine in the Treatment of Adolescent Idiopathic Scoliosis," The Archives of Bone and Joint Surgery, vol. 4, no. 4, pp. 376-380, 2016. DOI: 10.22038/abjs.2016.7538

[16] E. Ameri, H. Ghandhari, N. Nabizadeh and H. Hesarikia, "Natural history of coronal balance after spinal fusion in adolescent idiopathic scoliosis," Journal of Research in Orthopedic Science, vol. 1, no. 2, 2014. URL: http://jros.iums.ac.ir/article-1-117-en.html

[17] M. Palaniswamy and W. Y. Leong, "Evaluation on Thoracic Trunk Shift and Coronal balance in Post-Operative Scoliosis Patients," ASM Science Journal, vol. 11, no. 1, pp. 1-8, 2018. URL: https://www.akademisains.gov.my/asmsj/article/evaluation-on- 
Asian Journal of Medical Technology (AJMedTech)

thoracic-trunk-shift-and-coronal-balance-in-post-operative-scoliosispatients/ 